\title{
An Efficient Hydration and Tandem Transfer Hydrogenation of Alkynes for the Synthesis of Alcohol in Water
}

\author{
Nianhua Luo ${ }^{a}$ \\ Yuhong Zhong ${ }^{\mathrm{a}}$ \\ Ji-Tian Liub \\ Lu Ouyang*a \\ Renshi Luo*a \\ a School of Pharmaceutical Sciences, Gannan Medical University, \\ Ganzhou, 341000, Jiangxi Province, P. R. of China \\ oyl0327@163.com \\ luorenshi2010@163.com \\ ${ }^{b}$ Department of Medicinal Chemistry, Key Laboratory of Chemical \\ Biology (Ministry of Education), School of Pharmaceutical Sciences, \\ Shandong University, 44 West Culture Road, 250012 Jinan, \\ Shandong, P. R. of China
}

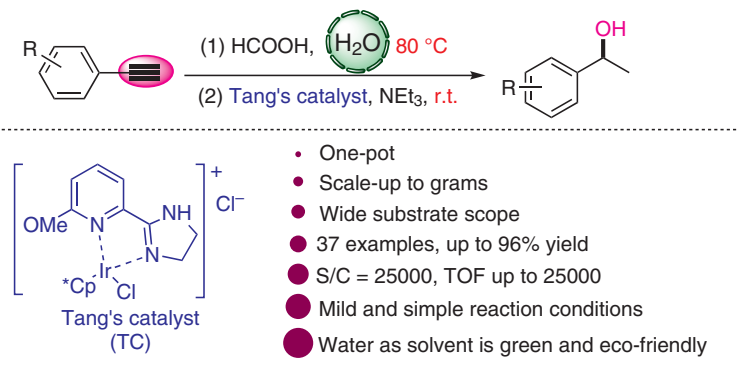

Water as solvent is green and eco-friendly

\begin{abstract}
Received: 21.05 .2020
Accepted after revision: 09.07.2020

Published online: 11.08 .2020

DOI: 10.1055/s-0040-1707233; Art ID: ss-2020-g0279-op

Abstract A practical and efficient method for the synthesis of alcohols in one pot from readily available alkynes via a tandem process by formic acid promoted hydration and metal-ligand bifunctional iridiumcatalyzed transfer hydrogenation under mild conditions has been described. This transformation is simple, efficient, and can be performed with a variety of alkynes in good yields and with excellent stereoselectivities. Experimental results showed high catalytic activity, and turnover frequency (TOF) up to 25000. Importantly, this transformation can be conducted in water, and is thus green and environmentally friendly.
\end{abstract}

Key words alkyne, hydration, transfer hydrogenation, metal-ligand bifunctional iridium

The functionalization of alkynes has an extremely significant position in the chemical industry, ${ }^{1}$ especially for the hydration of alkynes, because of the wide availability of alkynyl substrates and the great importance of the carbonyl motif in organic chemistry, and the atom-economy and pot-economy of the reaction. ${ }^{2}$ In contrast to traditional multistep synthetic procedures, pot-economic reactions are convergent, facile and efficient, and proceed with minimum isolation and purification. ${ }^{3}$ Thus, an interesting and promising synthetic method for the preparation of alcohols is the hydration of alkynes in one pot.

In the past decades, the one-pot synthesis of alcohols from alkynes via hydration/transfer hydrogenation has rarely been explored because of the incompatibility between the catalyst system and reaction conditions of the two steps. ${ }^{4}$ For example, in 2013, Xiao and co-workers reported a hydration and transfer hydrogenation of alkynes with formic acid-promoted hydration coupled with Ir-catalyzed transfer hydrogenation. ${ }^{5}$ However, the hydration process was conducted at high temperature $\left(100^{\circ} \mathrm{C}\right)$ and with an excess amount of formic acid as solvent. Furthermore, the catalytic transfer hydrogenation was not efficient, delivering alcohols in moderate yield under heating. Subsequently, examples for the synthesis of alcohols via one-pot sequential hydration and reduction of alkynes by using bimetal catalysts, ${ }^{6}$ such as $\mathrm{Co}-\mathrm{Ru},{ }^{7} \mathrm{Au}-\mathrm{Rh},{ }^{8}$ and $\mathrm{Co}-\mathrm{Rh}^{9}$ were reported. However, despite the encouraging and real progress that has been made, issues of catalytic efficiency, substrate compatibility, and simple operation can still be improved.

Based on the great interest in metal-ligand bifunctional iridium catalyst, ${ }^{10}$ we have recently developed the $\mathrm{pH}$-dependent chemoselective transfer hydrogenation of $\alpha, \beta$-unsaturated aldehydes ${ }^{11}$ and selective hydroxylation and alkoxylation of silanes (Scheme 1a). ${ }^{12}$ Inspired by this meaningful progress and on the basis of our earlier work, we envisioned that formic acid could not only serve as a promoter for the hydration of alkynes, but also donate hydrogen for the process of transfer hydrogenation. Herein, we present an efficient hydration/transfer hydrogenation of alkynes via formic acid-promoted hydration and metalligand bifunctional iridium catalyzed tandem transfer

(a) Our previous work on metal-ligand bifunctional iridium catalyst:

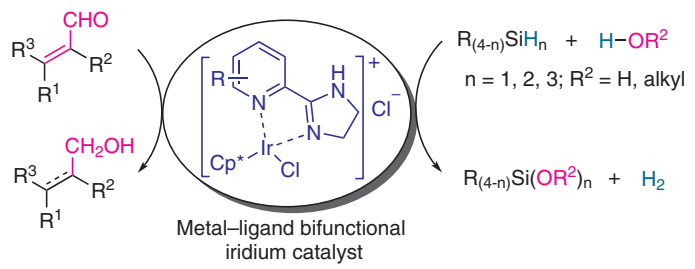

(b) This work:

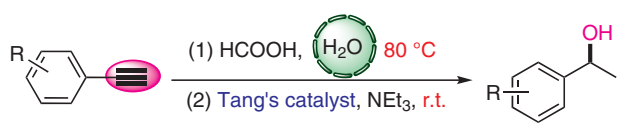

Scheme 1 One-pot conversion of alkynes into alcohols 
Table 1 Optimization of Catalyst for Hydration/Transfer Hydrogenation of Alkyne

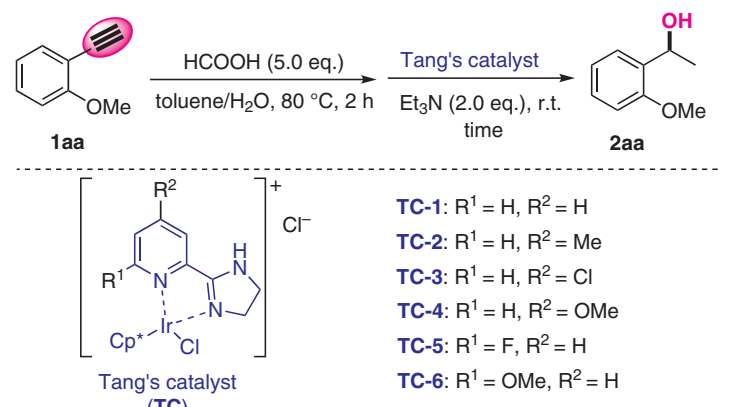

(TC)

\begin{tabular}{clcc}
\hline Entry & Catalyst (mol\%) & Reduction time (h) & ${\text { Yield }(\%)^{\mathrm{b}}}^{\mathrm{m}}$ \\
\hline 1 & TC-1 (1) & 6 & 94 \\
2 & TC-2 (1) & 8 & 97 \\
3 & TC-3 (1) & 10 & 68 \\
4 & TC-4 (1) & 2 & $>99$ \\
5 & TC-5 (1) & 10 & 83 \\
6 & TC-6 (1) & 0.2 & $>99(96)^{\mathrm{c}}$ \\
7 & TC-6 (0.1) & 0.5 & $>99$ \\
8 & TC-6 (0.05) & 10 & $>99$ \\
9 & TC-6 (0.01) & 16 & $>99$ \\
10 & - & 36 & not detected \\
\hline
\end{tabular}

a Reaction conditions: a mixture of 1 aa $(1.0 \mathrm{mmol})$, toluene $/ \mathrm{H}_{2} \mathrm{O}(1 \mathrm{~mL} / 1$ $\mathrm{mL}$ ), and formic acid (5.0 equiv) at $80{ }^{\circ} \mathrm{C}$ for $2 \mathrm{~h}$, then, $\mathrm{Et}_{3} \mathrm{~N}$ ( 2.0 equiv) and Tang's catalyst ( $1 \mathrm{~mol} \%$ ) at room temperature.

b Determined by GC-MS.

' Yield of isolated product.

hydrogenation to synthesize alcohols in high yield under mild reaction condition (Scheme $1 \mathrm{~b}$ ). The TOF is as high as 25000 , which is very efficient. In addition, this protocol relies on water as organic reaction media, which conforms with the concept of green and sustainable chemistry.

Preliminary investigation on the one-pot hydration/transfer hydrogenation of alkynes was conducted with 1-ethynyl-2-methoxybenzene (1a), formic acid, and Tang's catalyst (TC) ${ }^{13}$ (Table 1 ). First, the hydration reaction was conducted with 5.0 equiv $\mathrm{HCOOH}$ and $2 \mathrm{~mL}$ toluene $/ \mathrm{H}_{2} \mathrm{O}$ (1:1) as the solvent. To our delight, the hydration process performed well and the alkynes were completely converted into ketones in $80^{\circ} \mathrm{C}$ in 2 hours. Then, 2.0 equiv of $\mathrm{NEt}_{3}$ and $1 \mathrm{~mol} \%$ TC-1 were added to the reaction (entry 1 ). The transfer hydrogenation process proceeded at room temperature under air for $6 \mathrm{~h}$, giving the desired alcohols in $94 \%$ yield. Subsequently, exploration of different substituted catalysts established that TC-6 was the best choice for this transformation and the desired product 2 aa could be obtained in 96\% yield in $0.2 \mathrm{~h}$ (entries 2-6). In addition, to test the catalytic efficiency, catalyst loadings were screened. It is worth noting that $0.1 \mathrm{~mol} \%$ catalyst TC-6 exhibited high ef- ficiency, transforming the substrate completely into alcohols in 30 minutes (entries 7). It should be pointed out that none of the desired product was detected when the reaction was performed without metal catalyst (entry 10).

Given that the reaction medium can also affect the catalytic efficiency, other solvents were also surveyed (Table 2). Some were incompatible with the reaction, leading to lower yield. Surprisingly, we found that environmentally friendly water was the best reaction medium, and promoted the process more efficiently than other solvents.

With the optimal reaction conditions in hand, we next explored the scope of the sequential hydration/transfer hydrogenation process. A variety of alkynes with different substituents were explored and the results are summarized in Scheme 2. Substrates with either electron-donating groups (EDGs), such as methoxy and other alkyl moieties were tolerated well (2aa-ag, 2az-bb, 2bc-be), even in the sterically hindered ortho-position. In addition, substrates possessing a halogen group also reacted well, leading to the desired alcohols (2ah-ap, 2av-ax) in excellent yields. Furthermore, perfluorophenone or aromatic substrates substituted with electron-withdrawing groups (EWGs), such as $\mathrm{NO}_{2}, \mathrm{CF}_{3}$, and $\mathrm{CN}$, did not affect the process of hydration/transfer hydrogenation (2aq-au, 2ay). To our delight, we found that this protocol not only applied to terminal alkynes, but could also be used for internal alkynes. A range of internal alkynes all served well for this transformation (2bf, 2bg). Moreover, substrates with a higher degree of

Table 2 Optimization of Reaction Medium for Hydration/Transfer Hydrogenation of Alkyne

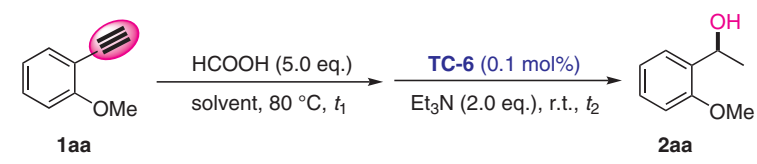

\begin{tabular}{|c|c|c|c|c|}
\hline Entry & Solvent & $\begin{array}{l}\text { Hydrolysis time } t_{1} \\
\text { (h) }\end{array}$ & $\begin{array}{l}\text { Reduction time } t_{2} \\
\text { (h) }\end{array}$ & $\begin{array}{l}\text { Yield } \\
(\%)^{\mathrm{b}}\end{array}$ \\
\hline 1 & toluene $/ \mathrm{H}_{2} \mathrm{O}$ & 2 & 0.5 & 96 \\
\hline 2 & hexane $/ \mathrm{H}_{2} \mathrm{O}$ & 16 & 6 & 74 \\
\hline 3 & $\mathrm{CH}_{2} \mathrm{Cl}_{2} / \mathrm{H}_{2} \mathrm{O}$ & 8 & 1 & 86 \\
\hline 4 & $\mathrm{Et}_{2} \mathrm{O} / \mathrm{H}_{2} \mathrm{O}$ & 12 & 4 & 88 \\
\hline 5 & acetone $/ \mathrm{H}_{2} \mathrm{O}$ & 12 & 2 & 85 \\
\hline 6 & $\mathrm{THF} / \mathrm{H}_{2} \mathrm{O}$ & 12 & 2 & 89 \\
\hline 7 & $\mathrm{DMF} / \mathrm{H}_{2} \mathrm{O}$ & 12 & 1 & 82 \\
\hline 8 & $\mathrm{MeOH} / \mathrm{H}_{2} \mathrm{O}$ & 16 & 4 & 78 \\
\hline $9^{c}$ & toluene & 24 & - & - \\
\hline $10^{d}$ & $\mathrm{H}_{2} \mathrm{O}$ & 2 & 0.5 & 96 \\
\hline \multicolumn{5}{|c|}{ 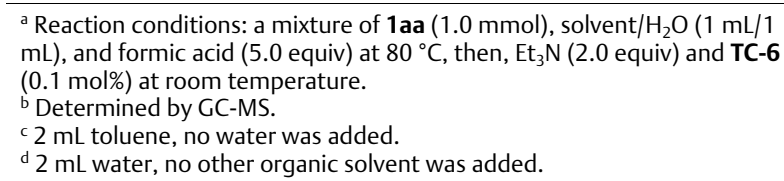 } \\
\hline
\end{tabular}


conjugation were amenable to this catalytic system (2bh, $\mathbf{2 b i}$ ). Interestingly, when heteroaromatic alkyne was treated under the standard conditions, the desired product $\mathbf{2} \mathbf{b j}$ could also be afforded in $94 \%$ yield. Furthermore, the alkyl alkyne cyclohexylacetylene was also successfully applied in this protocol (2bk).
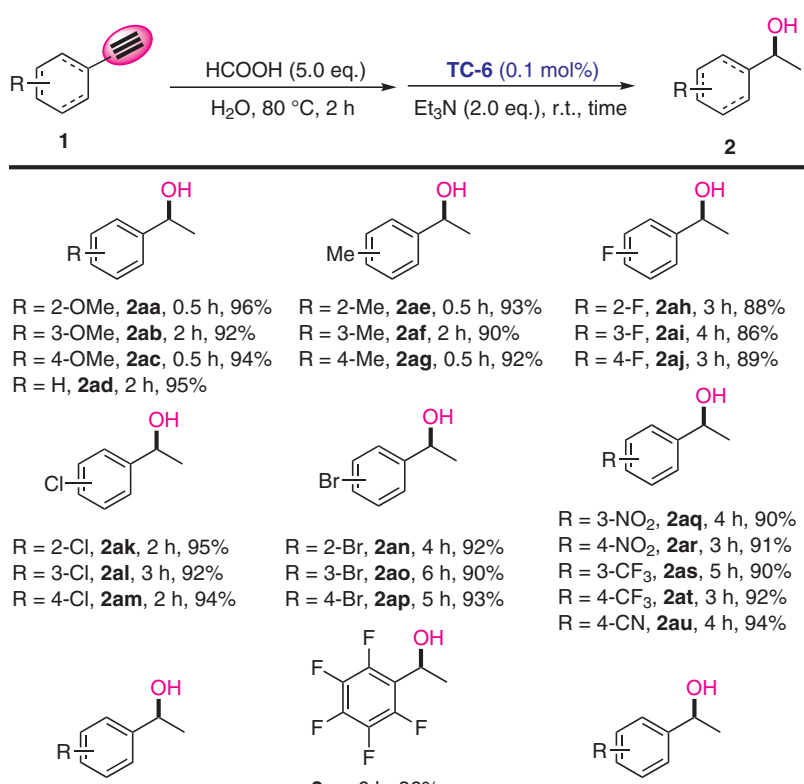

$\mathrm{R}=$ 3,4-dichloro, 2av, 2 h, $91 \%$ $\mathrm{R}=3$,4-difluoro, 2aw, 4 h, $92 \%$ $\mathrm{R}=3$-bromo-4-fluoro, 2ax, $6 \mathrm{~h}, 86 \%$

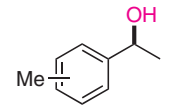

$\mathrm{R}=2-\mathrm{Me}, \mathbf{2 a e}, 0.5 \mathrm{~h}, 93 \%$ $\mathrm{R}=3-\mathrm{Me}, 2 \mathrm{af}, 2 \mathrm{~h}, 90 \%$

$\mathrm{R}=4-\mathrm{Me}, 2 \mathrm{ag}, 0.5 \mathrm{~h}, 92 \%$<smiles>CC(O)c1ccc(Br)cc1</smiles>

$\mathrm{R}=2-\mathrm{Br}, 2 \mathrm{an}, 4 \mathrm{~h}, 92 \%$ $\mathrm{R}=3-\mathrm{Br}, \mathbf{2 a o}, 6 \mathrm{~h}, 90 \%$ $\mathrm{R}=4-\mathrm{Br}$, 2ap, $5 \mathrm{~h}, 93 \%$<smiles>CC(O)c1c(F)c(F)c(F)c(F)c1F</smiles>
2ay, 6 h, $86 \%$

$\mathrm{R}=2$-methyl-5-hydroxy, 2az, 5 h, 90\% $\mathrm{R}=2$,3-dimethyl, 2 ba, $6 \mathrm{~h}, 92 \%$ $\mathrm{R}=2$-fluoro-5-hydroxy, 2bb, 4 h, $91 \%$

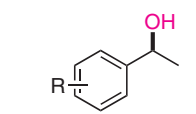

$\mathrm{R}=n$-butyl, 2bc, $6 \mathrm{~h}, 92 \%$ $\mathrm{R}=t$-butyl, 2bd, $6 \mathrm{~h}, 90 \%$ $\mathrm{R}=n$-hexyl, 2 be, $6 \mathrm{~h}, 90 \%$<smiles>CCC(O)c1ccccc1O</smiles>

2bj, 4 h, $94 \%$<smiles>CCCC(O)c1ccccc1</smiles>

2bg, 5 h, $92 \%$<smiles>CC(O)c1ccc2ccccc2c1</smiles>

2bi, 5 h, $91 \%$<smiles>CC(O)c1cccc2ccccc12</smiles>

2bh, 4 h, 95\%<smiles>CC(O)C1CCCCC1</smiles>

2bk, 5 h, $90 \%$
Scheme 2 Substrate scope of alkynes for hydration/transfer hydrogenation to synthesize alcohols. Reagents and conditions: a mixture of $\mathbf{1}$ (1.0 mmol), $\mathrm{H}_{2} \mathrm{O}(2 \mathrm{~mL})$, and formic acid (5.0 equiv) at $80{ }^{\circ} \mathrm{C}$ for $2 \mathrm{~h}$; then $\mathrm{Et}_{3} \mathrm{~N}$ ( 2.0 equiv) and TC-6 ( $0.1 \mathrm{~mol} \%$ ) at room temperature. Yield of isolated product given.

To examine the potential application of this hydration/transfer hydrogenation of alkynes, a gram-scale experiment (Scheme 3) and catalyst recycling efficiency studies (Table 3) were conducted. The scale-up experiment (1aa, 50 mmol) proceeded smoothly under the standard conditions with 0.004 mol\% catalyst loading, giving 2aa in 94\% yield, with a TOF as high as 25000 . We also found that metalligand bifunctional iridium catalysts could be recycled five times while maintaining high catalytic activity. Both results confirmed the great practical application of this sequential hydration/transfer hydrogenation transformation (Table 3).

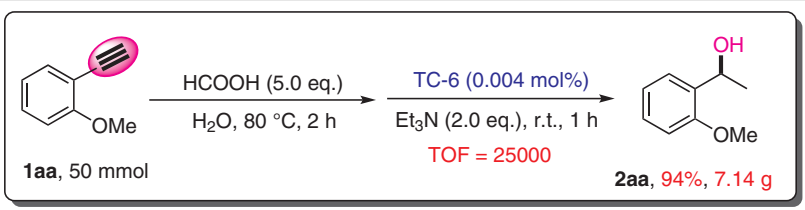

Scheme 3 Gram-scale hydration/transfer hydrogenation of 1 aa

Based on our experimental results and on previous reports, a possible mechanistic pathway for this hydration/transfer hydrogenation of alkynes is shown in Scheme 4. In the hydration step, the intermediate vinyl carbocation was obtained in the presence of $\mathrm{HCOOH}$, which allowed the hydration reaction to proceed smoothly under mild conditions. ${ }^{13,14}$ Then, transfer hydrogenation of ketones to alcohols proceeded with the aid of metal-ligand bifunctional iridium catalyst. ${ }^{15}$ Firstly, anion exchange occurs with formate anions to produce catalyst precursor $\mathbf{A}$, and carbon dioxide is extruded to generate active catalyst $\mathbf{B}$. The carbonyl is then activated via hydrogen bonding with hydrogen ions, which can lower the LUMO energy of carbonyl compounds and help the hydride addition to deliver intermediate $\mathbf{D} .^{16}$

Table 3 Catalytic Cycling Studies for the Transfer Hydrogenation ${ }^{\mathrm{a}}$

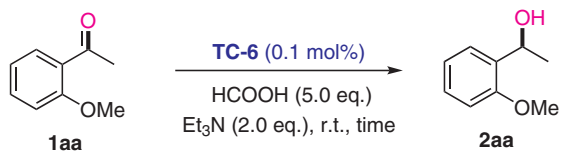

\begin{tabular}{lccccc}
\hline Cycle index & 1 & 2 & 3 & 4 & 5 \\
\hline Reaction time (h) & 0.5 & 0.5 & 1 & 1 & 1 \\
Yield $(\%)^{\mathrm{b}}$ & $>99$ & $>99$ & $>99$ & $>99$ & $>99$
\end{tabular}

a Reaction conditions: a mixture of 3 aa $(1.0 \mathrm{mmol}), \mathrm{H}_{2} \mathrm{O}(2 \mathrm{~mL})$, formic acid (5.0 equiv), $\mathrm{Et}_{3} \mathrm{~N}$ (2.0 equiv) and TC-6 (0.1 mol\%) at room temperature. b Determined by GC-MS.

(1) Hydration of alkynes:

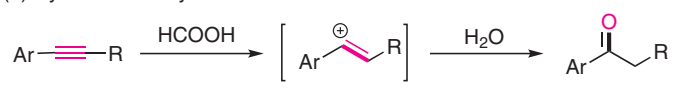

(2) Transfer hydrogenation of ketones via cyclometalated iridium catalyst:

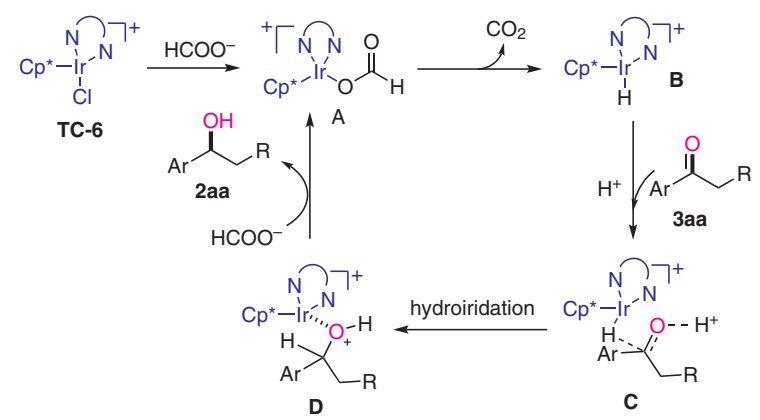

Scheme 4 Proposed mechanism for the hydration and transfer hydrogenation of alkynes 
Ligand exchange of intermediate $\mathbf{D}$ releases the desired products and catalyst precursor $\mathbf{A}$ for the next catalytic cycle.

In conclusion, we have developed an interesting method for the synthesis of alcohols in one pot from readily available alkynes by using a tandem process involving hydration and transfer hydrogenation. The simple operating procedure, mild reaction conditions, and high yields make this protocol particularly practical. The TOF is as high as 25000 , demonstrating the high catalytic activity. Most importantly, this transformation can be conducted in the aqueous phase, which is green and eco-friendly. Ongoing studies are focused on further exploring the asymmetric hydrogenation and the results will be reported in due course.

All reactions were performed under air in a dried flask. All solvents were purified by standard drying methods. Unless otherwise stated, commercial reagents were directly used without further purification. Products were purified by flash chromatography using silica gel (200300 mesh). ${ }^{1} \mathrm{H}$ NMR spectra were recorded on a Bruker 400 (400 $\mathrm{MHz}$ ) spectrometer with $\mathrm{CDCl} 3(\delta=7.26 \mathrm{ppm})$, or with tetramethylsilane (TMS, $\delta=0.00 \mathrm{ppm}$ ) as the internal standard. ${ }^{13} \mathrm{C}$ NMR spectra were were recorded on a Bruker $(100 \mathrm{MHz})$ spectrometer with $\mathrm{CDCl}_{3}$ as the internal reference $(\delta=77.0 \mathrm{ppm})$. The melting points were determined on WRR melting point apparatus and are uncorrected.

\section{Synthesis of Alcohols; General Procedure}

In a $10 \mathrm{~mL}$ Schlenk tube, formic acid (5.0 equiv) was added to a stirred solution of phenyl acetylene $\mathbf{1}(1.0 \mathrm{mmol})$ and $\mathrm{H}_{2} \mathrm{O}(2 \mathrm{~mL})$. The resulting suspension was vigorously stirred at $80{ }^{\circ} \mathrm{C}$ for $2 \mathrm{~h}$, then $\mathrm{Et}_{3} \mathrm{~N}(2.0$ equiv) and TC-6 (0.6 mg, $0.1 \mathrm{~mol} \%)$ dissolved in water were added and the mixture was stirred at r.t. until the completion of the reaction. The mixture was extracted with diethyl ether $(3 \times 10 \mathrm{~mL})$ and the combined diethyl ether layer was dried over sodium sulfate and concentrated in vacuum. After evaporation of the solvent, the crude mixture was purified by silica gel column chromatography (petroleum ether/EtOAc, 5:1) to afford the silanol product.

\section{Catalytic Cycle}

In a $10 \mathrm{~mL}$ Schlenk tube, a mixture of formic acid (5.0 equiv) and $\mathrm{Et}_{3} \mathrm{~N}$ (2.0 equiv) was added to a stirred solution of 1-(2-methoxyphenyl)ethanone (3aa; $1.0 \mathrm{mmol})$, TC-6 $(0.6 \mathrm{mg}, 0.1 \mathrm{~mol} \%)$ and $\mathrm{H}_{2} \mathrm{O}(2$ $\mathrm{mL})$ at r.t. After completion of the reaction, the mixture was extracted with EtOAc $(3 \times 10 \mathrm{~mL})$, the combined EtOAc layer was analyzed by GC-MS, and the water layer was reserved for the next cycle.

In a $10 \mathrm{~mL}$ Schlenk tube, a mixture of formic acid (5.0 equiv) and $\mathrm{Et}_{3} \mathrm{~N}$ (2.0 equiv) was added to a stirred solution of 1-(2-methoxyphenyl)ethanone (3aa; $1.0 \mathrm{mmol}$ ) in the water reserved in the last cycle at r.t. After completion of the reaction, the mixture was extracted with EtOAc $(3 \times 10 \mathrm{~mL})$, the combined EtOAc layer was analyzed by GC-MS, and the water layer was reserved for the next cycle.

\section{Gram-Scale Hydration/Transfer Hydrogenation}

In a $100 \mathrm{~mL}$ Schlenk tube, a mixture of formic acid (5.0 equiv) and $\mathrm{Et}_{3} \mathrm{~N}$ (2.0 equiv) was added to a stirred solution of 1-ethynyl-2-methoxybenzene (1aa; $50 \mathrm{mmol})$, TC-6 $(0.004 \mathrm{~mol} \%)$ and $\mathrm{H}_{2} \mathrm{O}(20 \mathrm{~mL})$ at r.t. After completion of the reaction, the mixture was extracted with EtOAc $(3 \times 100 \mathrm{~mL})$, and the combined EtOAc layer was dried over so- dium sulfate and concentrated in vacuum. After evaporation of the solvent, the crude mixture was purified by silica gel column chromatography to afford 2aa (7.14 g, 94\% yield).

\section{1-(2-Methoxyphenyl)ethanol (2aa) $)^{14 c}$}

Yield: $146.0 \mathrm{mg}$ (96\%); colorless oil.

${ }^{1} \mathrm{H}$ NMR (400 MHz, $\left.\mathrm{CDCl}_{3}\right): \delta=7.33(\mathrm{~d}, J=5.8 \mathrm{~Hz}, 1 \mathrm{H}), 7.20(\mathrm{~d}, J=$ $7.7 \mathrm{~Hz}, 1 \mathrm{H}), 6.92(\mathrm{~d}, J=6.9 \mathrm{~Hz}, 1 \mathrm{H}), 6.84(\mathrm{~d}, J=8.0 \mathrm{~Hz}, 1 \mathrm{H}), 5.07(\mathrm{~d}$, $J=5.3 \mathrm{~Hz}, 1 \mathrm{H}), 3.80(\mathrm{~s}, 3 \mathrm{H}), 2.97(\mathrm{~s}, 1 \mathrm{H}), 1.46(\mathrm{~d}, J=4.7 \mathrm{~Hz}, 3 \mathrm{H})$.

${ }^{13} \mathrm{C}$ NMR $\left(100 \mathrm{MHz}, \mathrm{CDCl}_{3}\right): \delta=156.4,133.6,128.2,126.1,120.8$, 110.4, 66.2, 55.3, 23.0.

\section{1-(3-Methoxyphenyl)ethanol (2ab) ${ }^{14 c}$}

Yield: $139.9 \mathrm{mg}$ (92\%); colorless oil.

${ }^{1} \mathrm{H}$ NMR $\left(400 \mathrm{MHz}, \mathrm{CDCl}_{3}\right): \delta=7.18(\mathrm{~d}, J=8.1 \mathrm{~Hz}, 1 \mathrm{H}), 6.94-6.82(\mathrm{~m}$, $2 \mathrm{H}), 6.78-6.70(\mathrm{~m}, 1 \mathrm{H}), 4.74(\mathrm{~d}, J=6.5 \mathrm{~Hz}, 1 \mathrm{H}), 3.72(\mathrm{~s}, 3 \mathrm{H}), 3.17$ (s, $1 \mathrm{H}), 1.39$ (d, $J=6.5 \mathrm{~Hz}, 3 \mathrm{H})$.

${ }^{13} \mathrm{C}$ NMR $\left(100 \mathrm{MHz}, \mathrm{CDCl}_{3}\right): \delta=159.6,147.8,129.5,117.8,112.7$, $111.0,70.1,55.2,25.2$.

\section{1-(4-Methoxyphenyl)ethanol (2ac) ${ }^{6 c}$}

Yield: $143.0 \mathrm{mg}$ (94\%); colorless oil.

${ }^{1} \mathrm{H}$ NMR $\left(400 \mathrm{MHz}, \mathrm{CDCl}_{3}\right): \delta=7.24(\mathrm{~d}, J=8.6 \mathrm{~Hz}, 2 \mathrm{H}), 6.89-6.79(\mathrm{~m}$, $2 \mathrm{H}), 4.77(\mathrm{q}, J=6.4 \mathrm{~Hz}, 1 \mathrm{H}), 3.76(\mathrm{~s}, 3 \mathrm{H}), 2.62(\mathrm{~s}, 1 \mathrm{H}), 1.42(\mathrm{~d}, J=$ $6.5 \mathrm{~Hz}, 3 \mathrm{H})$.

${ }^{13} \mathrm{C}$ NMR $\left(100 \mathrm{MHz}, \mathrm{CDCl}_{3}\right): \delta=158.9,138.1,126.7,113.8,69.8,55.3$, 25.1.

\section{1-Phenylethanol (2ad) ${ }^{14 \mathrm{c}}$}

Yield: $116.0 \mathrm{mg}$ (95\%); colorless oil.

${ }^{1} \mathrm{H}$ NMR $\left(400 \mathrm{MHz}, \mathrm{CDCl}_{3}\right): \delta=7.34-7.14(\mathrm{~m}, 5 \mathrm{H}), 4.74(\mathrm{q}, J=6.5 \mathrm{~Hz}$, $1 \mathrm{H}), 3.10(\mathrm{~s}, 1 \mathrm{H}), 1.38(\mathrm{~d}, J=6.5 \mathrm{~Hz}, 3 \mathrm{H})$.

${ }^{13} \mathrm{C}$ NMR $\left(100 \mathrm{MHz}, \mathrm{CDCl}_{3}\right): \delta=146.0,128.5,127.4,125.5,70.2,25.2$.

\section{1-o-Tolylethanol (2ae) ${ }^{7}$}

Yield: $126.6 \mathrm{mg}(93 \%)$; colorless oil.

${ }^{1} \mathrm{H}$ NMR $\left(400 \mathrm{MHz}, \mathrm{CDCl}_{3}\right): \delta=7.44(\mathrm{~d}, J=7.5 \mathrm{~Hz}, 1 \mathrm{H}), 7.20-7.07(\mathrm{~m}$, $3 \mathrm{H}), 5.01(\mathrm{q}, J=6.4 \mathrm{~Hz}, 1 \mathrm{H}), 2.54(\mathrm{~s}, 1 \mathrm{H}), 2.28(\mathrm{~s}, 3 \mathrm{H}), 1.38(\mathrm{~d}, J=$ $6.4 \mathrm{~Hz}, 3 \mathrm{H})$.

${ }^{13} \mathrm{C}$ NMR $\left(100 \mathrm{MHz}, \mathrm{CDCl}_{3}\right): \delta=144.0,134.2,130.4,127.1,126.4$, 124.6, 66.7, $23.95(\mathrm{~s}), 18.9$.

\section{1-m-Tolylethanol (2af)}

Yield: $122.5 \mathrm{mg}$ (90\%); colorless oil.

${ }^{1} \mathrm{H}$ NMR (400 MHz, $\left.\mathrm{CDCl}_{3}\right): \delta=7.13(\mathrm{t}, J=7.5 \mathrm{~Hz}, 1 \mathrm{H}), 7.08-7.00(\mathrm{~m}$, $2 \mathrm{H}), 6.97(\mathrm{~d}, J=7.5 \mathrm{~Hz}, 1 \mathrm{H}), 4.63(\mathrm{q}, J=6.4 \mathrm{~Hz}, 1 \mathrm{H}), 3.79(\mathrm{~s}, 1 \mathrm{H}), 2.26$ (s, $3 \mathrm{H}), 1.33(\mathrm{~d}, J=6.7 \mathrm{~Hz}, 3 \mathrm{H})$.

${ }^{13} \mathrm{C}$ NMR $\left(100 \mathrm{MHz}, \mathrm{CDCl}_{3}\right): \delta=146.2,138.0,128.5,128.4,128.4$, $128.1,126.4,122.7,70.1,25.3,21.6$.

\section{1-p-Tolylethanol (2ag) $)^{14 c}$}

Yield: $125.2 \mathrm{mg}$ (92\%); colorless oil.

${ }^{1} \mathrm{H}$ NMR $\left(400 \mathrm{MHz}, \mathrm{CDCl}_{3}\right): \delta=7.20(\mathrm{~d}, J=8.1 \mathrm{~Hz}, 2 \mathrm{H}), 7.11(\mathrm{~d}, J=$ $8.0 \mathrm{~Hz}, 2 \mathrm{H}), 4.76$ (q, J = 6.4 Hz, $1 \mathrm{H}), 2.58$ (s, $1 \mathrm{H}), 2.31$ (s, $3 \mathrm{H}), 1.41$ (d, $J=6.5 \mathrm{~Hz}, 3 \mathrm{H})$.

${ }^{13} \mathrm{C} \mathrm{NMR}\left(100 \mathrm{MHz}, \mathrm{CDCl}_{3}\right): \delta=143.0,137.0,129.2,125.5,25.2,21.2$. 


\section{1-(2-Fluorophenyl)ethanol (2ah) $)^{14 \mathrm{c}}$}

Yield: $123.3 \mathrm{mg}(88 \%)$; colorless oil.

${ }^{1} \mathrm{H}$ NMR $\left(400 \mathrm{MHz}, \mathrm{CDCl}_{3}\right): \delta=7.42-7.040(\mathrm{~m}, 1 \mathrm{H}), 7.19-7.17(\mathrm{~m}$, $1 \mathrm{H}), 7.10-7.06(\mathrm{~m}, 1 \mathrm{H}), 6.98-6.93(\mathrm{~m}, 1 \mathrm{H}), 5.11(\mathrm{q}, J=6.5 \mathrm{~Hz}, 1 \mathrm{H})$, $3.27(\mathrm{~s}, 1 \mathrm{H}), 1.42(\mathrm{~d}, J=6.5 \mathrm{~Hz}, 3 \mathrm{H})$.

${ }^{13} \mathrm{C}$ NMR $\left(100 \mathrm{MHz}, \mathrm{CDCl}_{3}\right): \delta=159.6(\mathrm{~d}, J=244 \mathrm{~Hz}), 132.8(\mathrm{~d}, J=$ $13 \mathrm{~Hz}), 128.7(\mathrm{~d}, J=5 \mathrm{~Hz}), 126.7(\mathrm{~d}, J=4 \mathrm{~Hz}), 124.3(\mathrm{~d}, J=3 \mathrm{~Hz}), 115.2$ (d, $J=21 \mathrm{~Hz}), 64.2(\mathrm{~d}, J=3 \mathrm{~Hz}), 24.0$.

${ }^{19} \mathrm{~F} \mathrm{NMR}\left(377 \mathrm{MHz}, \mathrm{CDCl}_{3}\right): \delta=-120.07(\mathrm{~s}, 1 \mathrm{~F})$.

\section{1-(3-Fluorophenyl)ethanol (2ai) ${ }^{6 c}$}

Yield: $120.5 \mathrm{mg}(86 \%)$; colorless oil.

${ }^{1} \mathrm{H} \mathrm{NMR}\left(400 \mathrm{MHz}, \mathrm{CDCl}_{3}\right): \delta=7.28-7.23(\mathrm{~m}, 1 \mathrm{H}), 7.07-7.01(\mathrm{~m}, 2 \mathrm{H})$, 6.94-6.89 (m, $1 \mathrm{H}), 4.78(\mathrm{q}, J=6.5 \mathrm{~Hz}, 1 \mathrm{H}), 3.19(\mathrm{~s}, 1 \mathrm{H}), 1.40(\mathrm{~d}, J=$ $6.5 \mathrm{~Hz}, 3 \mathrm{H})$.

${ }^{13} \mathrm{C}$ NMR $\left(100 \mathrm{MHz}, \mathrm{CDCl}_{3}\right): \delta=163.0(\mathrm{~d}, J=244 \mathrm{~Hz}), 148.6(\mathrm{~d}, J=$ $6 \mathrm{~Hz}), 130.0(\mathrm{~d}, J=8 \mathrm{~Hz}), 121.0(\mathrm{~d}, J=2 \mathrm{~Hz}), 114.1(\mathrm{~d}, J=21 \mathrm{~Hz}), 112.3$ $(\mathrm{d}, J=21 \mathrm{~Hz}), 69.6(\mathrm{~d}, J=2 \mathrm{~Hz}), 25.1$.

${ }^{19} \mathrm{~F}$ NMR $\left(377 \mathrm{MHz}, \mathrm{CDCl}_{3}\right): \delta=-112.95(\mathrm{~s}, 1 \mathrm{~F})$

\section{1-(4-Fluorophenyl)ethanol (2aj) $)^{14 c}$}

Yield: $124.7 \mathrm{mg}(89 \%)$; colorless oil.

$\left.{ }^{1} \mathrm{H} \mathrm{NMR} \mathrm{(400} \mathrm{MHz,} \mathrm{CDCl}_{3}\right): \delta=7.29-7.26(\mathrm{~m}, 2 \mathrm{H}), 7.02-6.98(\mathrm{~m}, 2 \mathrm{H})$, $4.78(\mathrm{q}, J=6.5 \mathrm{~Hz}, 1 \mathrm{H}), 3.35(\mathrm{~s}, 1 \mathrm{H}), 1.41(\mathrm{~d}, J=6.5 \mathrm{~Hz}, 3 \mathrm{H})$.

${ }^{13} \mathrm{C}$ NMR $\left(100 \mathrm{MHz}, \mathrm{CDCl}_{3}\right): \delta=162.0(\mathrm{~d}, J=244 \mathrm{~Hz}), 141.6(\mathrm{~d}, J=$ $3 \mathrm{~Hz}), 127.1$ (d, $J=8 \mathrm{~Hz}), 115.1(\mathrm{~d}, J=11 \mathrm{~Hz}), 69.5,25.2$.

${ }^{19} \mathrm{~F} \mathrm{NMR}\left(377 \mathrm{MHz}, \mathrm{CDCl}_{3}\right): \delta=-115.47(\mathrm{~s}, 1 \mathrm{~F})$

\section{1-(2-Chlorophenyl)ethanol (2ak) ${ }^{14 \mathrm{c}}$}

Yield: $148.2 \mathrm{mg}$ (95\%); colorless oil.

${ }^{1} \mathrm{H}$ NMR $\left(400 \mathrm{MHz}, \mathrm{CDCl}_{3}\right): \delta=7.50(\mathrm{dd}, J=7.7,1.7 \mathrm{~Hz}, 1 \mathrm{H}), 7.27-7.19$ $(\mathrm{m}, 2 \mathrm{H}), 7.15-7.12(\mathrm{~m}, 1 \mathrm{H}), 5.19(\mathrm{q}, J=6.4 \mathrm{~Hz}, 1 \mathrm{H}), 3.40(\mathrm{~s}, 1 \mathrm{H}), 1.38$ $(\mathrm{d}, J=6.5 \mathrm{~Hz}, 3 \mathrm{H})$.

${ }^{13} \mathrm{C}$ NMR $\left(100 \mathrm{MHz}, \mathrm{CDCl}_{3}\right): \delta=143.2,131.5,129.3,128.3,127.2$, $126.5,66.8,23.6$.

\section{1-(3-Chlorophenyl)ethanol (2al) $)^{5}$}

Yield: $143.5 \mathrm{mg}(92 \%)$; colorless oil.

${ }^{1} \mathrm{H} \mathrm{NMR}\left(400 \mathrm{MHz}, \mathrm{CDCl}_{3}\right): \delta=7.27(\mathrm{br}, 1 \mathrm{H}), 7.19-7.17(\mathrm{~m}, 2 \mathrm{H}), 7.12-$ $7.10(\mathrm{~m}, 1 \mathrm{H}), 4.69(\mathrm{q}, J=6.5 \mathrm{~Hz}, 1 \mathrm{H}), 3.81(\mathrm{~s}, 1 \mathrm{H}), 1.34(\mathrm{~d}, J=6.6 \mathrm{~Hz}$, $3 \mathrm{H})$.

${ }^{13} \mathrm{C}$ NMR $\left(100 \mathrm{MHz}, \mathrm{CDCl}_{3}\right): \delta=147.9,129.8,127.4,125.7,123.7,69.5$, 25.1 .

\section{1-(4-Chlorophenyl)ethanol (2am) $)^{14 c}$}

Yield: $146.6 \mathrm{mg}(94 \%)$; colorless oil.

${ }^{1} \mathrm{H}$ NMR $\left(400 \mathrm{MHz}, \mathrm{CDCl}_{3}\right): \delta=7.28-7.22(\mathrm{~m}, 4 \mathrm{H}), 4.78(\mathrm{q}, J=6.5 \mathrm{~Hz}$, $1 \mathrm{H}), 2.83(\mathrm{~s}, 1 \mathrm{H}), 1.40(\mathrm{~d}, J=6.5 \mathrm{~Hz}, 3 \mathrm{H})$.

${ }^{13} \mathrm{C} \mathrm{NMR}\left(100 \mathrm{MHz}, \mathrm{CDCl}_{3}\right): \delta=144.3,133.0,128.6,126.8,69.6,25.2$.

\section{1-(2-Bromophenyl)ethanol (2an) $)^{14 \mathrm{c}}$}

Yield: $184.0 \mathrm{mg}(92 \%)$; colorless oil.

${ }^{1} \mathrm{H}$ NMR (400 MHz, $\mathrm{CDCl}_{3}$ ): $\delta=7.51-7.44(\mathrm{~m}, 2 \mathrm{H}), 7.29-7.25(\mathrm{~m}, 1 \mathrm{H})$, 7.08-7.04 (m, $1 \mathrm{H}), 5.14(\mathrm{q}, J=6.4 \mathrm{~Hz}, 1 \mathrm{H}), 3.28(\mathrm{~s}, 1 \mathrm{H}), 1.39(\mathrm{~d}, J=$ $6.4 \mathrm{~Hz}, 3 \mathrm{H})$
${ }^{13} \mathrm{C}$ NMR $\left(100 \mathrm{MHz}, \mathrm{CDCl}_{3}\right): \delta=144.8,132.6,128.7,127.9,126.8$, 121.6, 69.1, 23.7.

\section{1-(3-Bromophenyl)ethanol (2ao) $)^{14 \mathrm{c}}$}

Yield: $180.0 \mathrm{mg}$ (90\%); colorless oil.

${ }^{1} \mathrm{H}$ NMR (400 MHz, $\mathrm{CDCl}_{3}$ ): $\delta=7.45$ (br $1 \mathrm{H}$ ), 7.36-7.33 (m, $1 \mathrm{H}$ ), 7.20$7.13(\mathrm{~m}, 2 \mathrm{H}), 4.72(\mathrm{q}, J=6.5 \mathrm{~Hz}, 1 \mathrm{H}), 3.38(\mathrm{~s}, 1 \mathrm{H}), 1.37$ (d, $J=6.5 \mathrm{~Hz}$, $3 \mathrm{H})$.

${ }^{13} \mathrm{C}$ NMR $\left(100 \mathrm{MHz}, \mathrm{CDCl}_{3}\right): \delta=148.2,130.4,130.1,128.6,124.1$, $122.5,69.5,25.2$.

\section{1-(4-Bromophenyl)ethanol (2ap) $)^{14 c}$}

Yield: $186.0 \mathrm{mg}$ (93\%); colorless oil.

${ }^{1} \mathrm{H}$ NMR $\left(400 \mathrm{MHz}, \mathrm{CDCl}_{3}\right): \delta=7.42(\mathrm{~d}, J=8.5 \mathrm{~Hz}, 2 \mathrm{H}), 7.17(\mathrm{~d}, J=$ $8.3 \mathrm{~Hz}, 2 \mathrm{H}), 4.76$ (q, $J=6.5 \mathrm{~Hz}, 1 \mathrm{H}), 2.85$ (s, $1 \mathrm{H}), 1.39$ (d, $J=6.5 \mathrm{~Hz}$, $3 \mathrm{H})$.

${ }^{13} \mathrm{C}$ NMR $\left(100 \mathrm{MHz}, \mathrm{CDCl}_{3}\right): \delta=144.8,131.5,127.2,121.1,69.6,25.2$.

\section{1-(3-Nitrophenyl)ethanol (2aq) $)^{17 d}$}

Yield: $150.4 \mathrm{mg}$ (90\%); colorless oil.

${ }^{1} \mathrm{H}$ NMR $\left(400 \mathrm{MHz}, \mathrm{CDCl}_{3}\right): \delta=8.18(\mathrm{~s}, 1 \mathrm{H}), 8.04(\mathrm{br} 1 \mathrm{H}), 7.69(\mathrm{~d}, J=$ $7.7 \mathrm{~Hz}, 1 \mathrm{H}), 7.49(\mathrm{t}, J=7.9 \mathrm{~Hz}, 1 \mathrm{H}), 4.99(\mathrm{q}, J=6.5 \mathrm{~Hz}, 1 \mathrm{H}), 4.20(\mathrm{~s}$, $1 \mathrm{H}), 1.49(\mathrm{~d}, J=6.6 \mathrm{~Hz}, 3 \mathrm{H})$.

${ }^{13} \mathrm{C} \mathrm{NMR}\left(100 \mathrm{MHz}, \mathrm{CDCl}_{3}\right): \delta=148.0,131.8,129.4,122.1,120.2,69.1$, 25.2 .

\section{1-(4-Nitrophenyl)ethanol (2ar) $)^{14 c}$}

Yield: $152.1 \mathrm{mg}$ (91\%); colorless oil.

${ }^{1} \mathrm{H}$ NMR $\left(400 \mathrm{MHz}, \mathrm{CDCl}_{3}\right): \delta=8.13(\mathrm{~d}, J=8.7 \mathrm{~Hz}, 2 \mathrm{H}), 7.52(\mathrm{~d}, J=$ $8.7 \mathrm{~Hz}, 2 \mathrm{H}), 5.00$ (q, J = 6.5 Hz, $1 \mathrm{H}), 3.38(\mathrm{~s}, 1 \mathrm{H}), 1.49$ (d, J = 6.6 Hz, $3 \mathrm{H})$.

${ }^{13} \mathrm{C}$ NMR $\left(100 \mathrm{MHz}, \mathrm{CDCl}_{3}\right): \delta=153.4,146.9,126.2,123.6,69.3,25.3$.

\section{1-(3-Trifluoromethylphenyl)ethanol (2as) ${ }^{14 \mathrm{c}}$}

Yield: $171.1 \mathrm{mg}$ (90\%); colorless oil.

${ }^{1} \mathrm{H}$ NMR $\left(400 \mathrm{MHz}, \mathrm{CDCl}_{3}\right): \delta=7.59(\mathrm{~s}, 1 \mathrm{H}), 7.50-7.39(\mathrm{~m}, 3 \mathrm{H}), 4.83$ (q, $J=6.4 \mathrm{~Hz}, 1 \mathrm{H}), 3.46(\mathrm{~s}, 1 \mathrm{H}), 1.41(\mathrm{~d}, J=6.5 \mathrm{~Hz}, 3 \mathrm{H})$.

${ }^{13} \mathrm{C}$ NMR $\left(100 \mathrm{MHz}, \mathrm{CDCl}_{3}\right): \delta=146.7,130.7(\mathrm{q}, J=32.1 \mathrm{~Hz}), 128.9$, $128.8,124.10(\mathrm{q}, J=3.7 \mathrm{~Hz}), 122.14(\mathrm{q}, J=3.7 \mathrm{~Hz}), 69.6,25.1$.

${ }^{19} \mathrm{~F}$ NMR $\left(377 \mathrm{MHz}, \mathrm{CDCl}_{3}\right): \delta=-62.59(\mathrm{~s}, 3 \mathrm{~F})$.

\section{1-(4-Trifluoromethylphenyl)ethanol (2at) ${ }^{14 c}$}

Yield: $174.9 \mathrm{mg}$ (92\%); colorless oil.

${ }^{1} \mathrm{H}$ NMR (400 MHz, $\left.\mathrm{CDCl}_{3}\right): \delta=7.57(\mathrm{~d}, J=8.1 \mathrm{~Hz}, 2 \mathrm{H}), 7.42(\mathrm{~d}, J=$ $8.4 \mathrm{~Hz}, 2 \mathrm{H}), 4.87$ (q, $J=6.5 \mathrm{~Hz}, 1 \mathrm{H}), 2.92(\mathrm{~s}, 1 \mathrm{H}), 1.44(\mathrm{~d}, J=6.5 \mathrm{~Hz}$, $3 \mathrm{H})$.

${ }^{13} \mathrm{C}$ NMR (100 MHz, $\left.\mathrm{CDCl}_{3}\right): \delta=149.7,129.6(\mathrm{q}, J=32.5 \mathrm{~Hz}), 125.6$, $125.4(\mathrm{q}, J=3.7 \mathrm{~Hz}), 124.2(\mathrm{q}, J=271.9 \mathrm{~Hz}), 69.7,25.2$.

${ }^{19} \mathrm{~F}$ NMR (377 MHz, $\left.\mathrm{CDCl}_{3}\right): \delta=-105.27(\mathrm{~s}, 3 \mathrm{~F})$

\section{4-(1-Hydroxyethyl)benzonitrile (2au) ${ }^{14 c}$}

Yield: $138.3 \mathrm{mg}$ (94\%); colorless oil.

${ }^{1} \mathrm{H}$ NMR $\left(400 \mathrm{MHz}, \mathrm{CDCl}_{3}\right): \delta=7.58(\mathrm{~d}, J=8.4 \mathrm{~Hz}, 2 \mathrm{H}), 7.47(\mathrm{~d}, J=$ $8.2 \mathrm{~Hz}, 2 \mathrm{H}), 4.91(\mathrm{q}, J=6.5 \mathrm{~Hz}, 1 \mathrm{H}), 3.45(\mathrm{~s}, 1 \mathrm{H}), 1.46(\mathrm{~d}, J=6.6 \mathrm{~Hz}$, $3 \mathrm{H})$. 
${ }^{13} \mathrm{C}$ NMR $\left(100 \mathrm{MHz}, \mathrm{CDCl}_{3}\right): \delta=151.5,132.2,126.1,118.9,110.5,69.3$, 25.3.

\section{1-(3,4-Dichlorophenyl)ethanol (2av)}

Yield: $171.0 \mathrm{mg}(90 \%)$; colorless oil.

${ }^{1} \mathrm{H} \mathrm{NMR}\left(400 \mathrm{MHz}, \mathrm{CDCl}_{3}\right): \delta=7.38(\mathrm{dd}, J=17.4,5.1 \mathrm{~Hz}, 2 \mathrm{H}), 7.12$ (dd, $J=8.3,2.0 \mathrm{~Hz}, 1 \mathrm{H}), 4.77(\mathrm{q}, J=6.5 \mathrm{~Hz}, 1 \mathrm{H}), 3.04(\mathrm{~s}, 1 \mathrm{H}), 1.41(\mathrm{~d}, J=$ $6.5 \mathrm{~Hz}, 3 \mathrm{H})$.

${ }^{13} \mathrm{C}$ NMR $\left(100 \mathrm{MHz}, \mathrm{CDCl}_{3}\right): \delta=146.0,132.4,131.1,130.4,127.4$, $124.8,69.1,25.2$.

\section{1-(3,4-Difluorophenyl)ethanol (2aw) $)^{17 \mathrm{e}}$}

Yield: $143.9 \mathrm{mg}(91 \%)$; colorless oil.

${ }^{1} \mathrm{H}$ NMR (400 MHz, $\left.\mathrm{CDCl}_{3}\right): \delta=7.22-6.92(\mathrm{~m}, 3 \mathrm{H}), 4.79$ (q, $J=6.4 \mathrm{~Hz}$, $1 \mathrm{H}), 2.91(\mathrm{~s}, 1 \mathrm{H}), 1.41(\mathrm{~d}, J=6.5 \mathrm{~Hz}, 3 \mathrm{H})$.

${ }^{13} \mathrm{C}$ NMR $\left(100 \mathrm{MHz}, \mathrm{CDCl}_{3}\right): \delta=150.2(\mathrm{dd}, J=241,12 \mathrm{~Hz}), 149.4(\mathrm{dd}$, $J=264,12 \mathrm{~Hz}), 142.9(\mathrm{t}, J=4 \mathrm{~Hz}), 121.3(\mathrm{q}, J=4 \mathrm{~Hz}), 117.1(\mathrm{~d}, J=$ $17 \mathrm{~Hz}), 114.3(\mathrm{~d}, J=18 \mathrm{~Hz}), 69.2,25.2$.

${ }^{19} \mathrm{~F} \mathrm{NMR}\left(377 \mathrm{MHz}, \mathrm{CDCl}_{3}\right): \delta=-137.72(\mathrm{~d}, J=8.8 \mathrm{~Hz}, 1 \mathrm{~F}),-140.16(\mathrm{~d}$, $J=11.0 \mathrm{~Hz}, 2 \mathrm{~F})$.

\section{1-(3-Bromo-4-fluorophenyl)ethanol (2ax) $)^{17 \mathrm{c}}$}

Yield: $200.6 \mathrm{mg}$ (92\%); colorless oil.

${ }^{1} \mathrm{H}$ NMR $\left(400 \mathrm{MHz}, \mathrm{CDCl}_{3}\right): \delta=7.52(\mathrm{dd}, J=6.6,2.1 \mathrm{~Hz}, 1 \mathrm{H}), 7.23-7.19$ $(\mathrm{m}, 1 \mathrm{H}), 7.05(\mathrm{t}, J=8.4 \mathrm{~Hz}, 1 \mathrm{H}), 4.78(\mathrm{q}, J=6.4 \mathrm{~Hz}, 1 \mathrm{H}), 2.98(\mathrm{~s}, 1 \mathrm{H})$, $1.41(\mathrm{~d}, J=6.5 \mathrm{~Hz}, 3 \mathrm{H})$.

${ }^{13} \mathrm{C}$ NMR $\left(100 \mathrm{MHz}, \mathrm{CDCl}_{3}\right): \delta=158.2(\mathrm{~d}, J=245 \mathrm{~Hz}), 143.1(\mathrm{~d}, J=$ $4 \mathrm{~Hz}), 130.5,126.0$ (d, $J=7 \mathrm{~Hz}), 116.3$ (d, $J=22 \mathrm{~Hz}), 108.9$ (d, $J=$ $20 \mathrm{~Hz}), 69.1,25.3$.

${ }^{19} \mathrm{~F} \mathrm{NMR}\left(377 \mathrm{MHz}, \mathrm{CDCl}_{3}\right): \delta=-62.39(\mathrm{~s}, 1 \mathrm{~F})$.

\section{1-Pentafluorophenylethanol (2ay) ${ }^{17 d}$}

Yield: $182.3 \mathrm{mg}(86 \%)$; colorless oil.

${ }^{1} \mathrm{H}$ NMR (400 MHz, $\mathrm{CDCl}_{3}$ ): $\delta=5.25$ (p, $\left.J=6.8 \mathrm{~Hz}, 1 \mathrm{H}\right), 2.57(\mathrm{~d}, J=$ $7.3 \mathrm{~Hz}, 1 \mathrm{H}), 1.65(\mathrm{~d}, J=6.8 \mathrm{~Hz}, 3 \mathrm{H})$.

${ }^{13} \mathrm{C}$ NMR $\left(100 \mathrm{MHz}, \mathrm{CDCl}_{3}\right): \delta=132.4,131.5,130.5,130.0,127.7$, $126.8,122.4,57.8,18.1$.

${ }^{19} \mathrm{~F}$ NMR (377 MHz, $\mathrm{CDCl}_{3}$ ): $\delta=-105.34(\mathrm{~d}, J=3.4 \mathrm{~Hz}, 2 \mathrm{~F}),-129.90$ $(\mathrm{dd}, J=20.9,3.9 \mathrm{~Hz}, 2 \mathrm{~F}),-136.19(\mathrm{dd}, J=20.9,3.4 \mathrm{~Hz}, 1 \mathrm{~F})$.

\section{3-(1-Hydroxyethyl)-4-methylphenol (2az)}

Yield: $136.9 \mathrm{mg}(90 \%)$; colorless oil.

${ }^{1} \mathrm{H}$ NMR (400 MHz, $\left.\mathrm{CDCl}_{3}\right): \delta=8.02(\mathrm{~s}, 1 \mathrm{H}), 6.92-6.90(\mathrm{~m}, 1 \mathrm{H}), 6.75-$ $6.70(\mathrm{~m}, 2 \mathrm{H}), 4.93(\mathrm{q}, J=6.6 \mathrm{~Hz}, 1 \mathrm{H}), 3.57(\mathrm{~s}, 1 \mathrm{H}), 2.22(\mathrm{~s}, 3 \mathrm{H}), 1.50$ $(\mathrm{d}, J=6.6 \mathrm{~Hz}, 3 \mathrm{H})$.

${ }^{13} \mathrm{C}$ NMR $\left(100 \mathrm{MHz}, \mathrm{CDCl}_{3}\right): \delta=152.6,129.3,129.2,128.6,127.1$, 116.7, 71.0, 23.4, 20.5.

HRMS-ESI: $m / z$ [M - $\mathrm{H}]^{-}$calcd for $\mathrm{C}_{9} \mathrm{H}_{12} \mathrm{O}_{2}$ : 151.0765; found: 151.0771 .

\section{1-(2,5-Dimethylphenyl)ethanol (2ba) $)^{17 b}$}

Yield: $138.1 \mathrm{mg}(92 \%)$; colorless oil.

${ }^{1} \mathrm{H}$ NMR $\left(400 \mathrm{MHz}, \mathrm{CDCl}_{3}\right): \delta=7.25(\mathrm{~s}, 1 \mathrm{H}), 6.95-6.89(\mathrm{~m}, 2 \mathrm{H}), 4.93$ (q, $J=6.4 \mathrm{~Hz}, 1 \mathrm{H}), 2.94(\mathrm{~s}, 1 \mathrm{H}), 2.27(\mathrm{~s}, 3 \mathrm{H}), 2.20(\mathrm{~s}, 3 \mathrm{H}), 1.34(\mathrm{~d}, J=$ $6.5 \mathrm{~Hz}, 3 \mathrm{H})$.
${ }^{13} \mathrm{C}$ NMR $\left(100 \mathrm{MHz}, \mathrm{CDCl}_{3}\right): \delta=143.9,135.7,131.0,130.3,127.8$, 125.4, 66.7, 24.0, 21.2, 18.5.

\section{4-Fluoro-3-(1-hydroxyethyl)phenol (2bb)}

Yield: $142.1 \mathrm{mg}(91 \%)$; colorless oil.

${ }^{1} \mathrm{H} \mathrm{NMR}\left(400 \mathrm{MHz}, \mathrm{CDCl}_{3}\right): \delta=8.01(\mathrm{~d}, J=31.2 \mathrm{~Hz}, 1 \mathrm{H}), 6.87-6.69(\mathrm{~m}$, $3 \mathrm{H}), 4.99(\mathrm{q}, J=6.6 \mathrm{~Hz}, 1 \mathrm{H}), 3.26(\mathrm{~s}, 1 \mathrm{H}), 1.54(\mathrm{~d}, J=6.6 \mathrm{~Hz}, 3 \mathrm{H})$.

${ }^{13} \mathrm{C}$ NMR $\left(100 \mathrm{MHz}, \mathrm{CDCl}_{3}\right): \delta=156.5(\mathrm{~d}, J=237.7 \mathrm{~Hz}), 151.0(\mathrm{~d}, J=$ $1.9 \mathrm{~Hz}), 129.7(\mathrm{~d}, J=6.4 \mathrm{~Hz}), 117.7(\mathrm{~d}, J=7.9 \mathrm{~Hz}), 115.0(\mathrm{~d}, J=22.9 \mathrm{~Hz})$, $113.0(\mathrm{~d}, J=23.6 \mathrm{~Hz}), 70.6,23.2$.

${ }^{19} \mathrm{~F}$ NMR (377 MHz, $\mathrm{CDCl}_{3}$ ): $\delta=-123.97(\mathrm{~s}, 1 \mathrm{~F})$.

HRMS-ESI: $m / z\left[M-\mathrm{H}^{-}\right.$calcd for $\mathrm{C}_{8} \mathrm{H}_{9} \mathrm{O}_{2} \mathrm{~F}$ : 155.0514; found: 155.0518 .

\section{1-(4-Butylphenyl)ethanol (2bc) ${ }^{17 \mathrm{f}}$}

Yield: $163.9 \mathrm{mg}$ (92\%); colorless oil.

${ }^{1} \mathrm{H}$ NMR $\left(400 \mathrm{MHz}, \mathrm{CDCl}_{3}\right): \delta=7.19(\mathrm{~d}, J=8.1 \mathrm{~Hz}, 2 \mathrm{H}), 7.10(\mathrm{~d}, J=$ $8.0 \mathrm{~Hz}, 2 \mathrm{H}), 4.72(\mathrm{q}, J=6.4 \mathrm{~Hz}, 1 \mathrm{H}), 3.03(\mathrm{~s}, 1 \mathrm{H}), 258-2.54(\mathrm{~m}, 2 \mathrm{H})$, 1.61-1.53 (m, $2 \mathrm{H}), 1.38-1.31(\mathrm{~m}, 5 \mathrm{H}), 0.91(\mathrm{t}, J=7.4 \mathrm{~Hz}, 3 \mathrm{H})$.

${ }^{13} \mathrm{C} \mathrm{NMR}\left(100 \mathrm{MHz}, \mathrm{CDCl}_{3}\right): \delta=143.3,142.0,128.5,125.5,125.5,70.1$, $35.4,33.8,25.1,22.5,14.1$.

\section{1-(4-tert-Butylphenyl)ethanol (2bd) ${ }^{7}$}

Yield: $160.3 \mathrm{mg}$ (90\%); white solid; mp $64-65^{\circ} \mathrm{C}$.

${ }^{1} \mathrm{H}$ NMR (400 MHz, $\mathrm{CDCl}_{3}$ ): $\delta=7.33(\mathrm{~d}, J=8.3 \mathrm{~Hz}, 2 \mathrm{H}), 7.24(\mathrm{~d}, J=$ $8.3 \mathrm{~Hz}, 2 \mathrm{H}$ ), 4.76 (q, $J=6.4 \mathrm{~Hz}, 1 \mathrm{H}), 2.75(\mathrm{~s}, 1 \mathrm{H}), 1.41$ (d, $J=6.5 \mathrm{~Hz}$, $3 \mathrm{H}), 1.30(\mathrm{~s}, 9 \mathrm{H})$

${ }^{13} \mathrm{C}$ NMR $\left(100 \mathrm{MHz}, \mathrm{CDCl}_{3}\right): \delta=150.3,143.0,125.4,125.3,70.0,34.6$, $31.5,25.0$.

\section{1-(4-Hexylphenyl)ethanol (2be) ${ }^{17 a}$}

Yield: $185.6 \mathrm{mg}$ (90\%); colorless oil.

${ }^{1} \mathrm{H}$ NMR $\left(400 \mathrm{MHz}, \mathrm{CDCl}_{3}\right): \delta=7.26(\mathrm{~d}, J=8.0 \mathrm{~Hz}, 2 \mathrm{H}), 7.15(\mathrm{~d}, J=$ $8.0 \mathrm{~Hz}, 2 \mathrm{H}), 4.83(\mathrm{q}, J=6.4 \mathrm{~Hz}, 1 \mathrm{H}), 2.60-2.56(\mathrm{~m}, 2 \mathrm{H}), 2.12(\mathrm{~s}, 1 \mathrm{H})$, $1.61-1.57(\mathrm{~m}, 2 \mathrm{H}), 1.46(\mathrm{~d}, J=6.5 \mathrm{~Hz}, 3 \mathrm{H}), 1.33-1.28(\mathrm{~m}, 6 \mathrm{H}), 0.88(\mathrm{t}$, $J=6.6 \mathrm{~Hz}, 3 \mathrm{H})$.

${ }^{13} \mathrm{C}$ NMR $\left(100 \mathrm{MHz}, \mathrm{CDCl}_{3}\right): \delta=143.1,142.3,128.5,125.4,70.3,35.7$, $31.8,31.6,29.1,25.0,22.7,14.2$.

\section{2-(1-Hydroxypropyl)phenol (2bf)}

Yield: $143.0 \mathrm{mg}$ (94\%); colorless oil.

${ }^{1} \mathrm{H} \mathrm{NMR}\left(400 \mathrm{MHz}, \mathrm{CDCl}_{3}\right): \delta=8.28(\mathrm{~s}, 1 \mathrm{H}), 7.10$ (br 1 H), 6.91-6.89 $(\mathrm{m}, 1 \mathrm{H}), 6.81-6.78(\mathrm{~m}, 2 \mathrm{H}), 4.64(\mathrm{t}, J=6.8 \mathrm{~Hz}, 1 \mathrm{H}), 3.72(\mathrm{~s}, 1 \mathrm{H})$, $1.83-1.77(\mathrm{~m}, 2 \mathrm{H}), 0.89(\mathrm{t}, J=7.4 \mathrm{~Hz}, 3 \mathrm{H})$.

${ }^{13} \mathrm{C}$ NMR $\left(100 \mathrm{MHz}, \mathrm{CDCl}_{3}\right): \delta=155.1,128.8,127.6,127.5,119.9$, 116.9, 76.9, 30.2, 10.2.

HRMS-ESI: $m / z$ [M - $\mathrm{H}]^{-}$calcd for $\mathrm{C}_{9} \mathrm{H}_{12} \mathrm{O}_{2}:$ 151.0765; found: 151.0766

\section{1-Phenyl-butan-1-ol (2bg) ${ }^{6 c}$}

Yield: $138.1 \mathrm{mg}$ (92\%); colorless oil.

${ }^{1} \mathrm{H}$ NMR $\left(400 \mathrm{MHz}, \mathrm{CDCl}_{3}\right): \delta=7.34-7.18(\mathrm{~m}, 5 \mathrm{H}), 4.61-4.49(\mathrm{~m}, 1 \mathrm{H})$, $2.63(\mathrm{~s}, 1 \mathrm{H}), 1.73-1.58(\mathrm{~m}, 2 \mathrm{H}), 1.38-1.23(\mathrm{~m}, 2 \mathrm{H}), 0.89(\mathrm{t}, J=7.4 \mathrm{~Hz}$, $3 \mathrm{H})$.

${ }^{13} \mathrm{C}$ NMR $\left(100 \mathrm{MHz}, \mathrm{CDCl}_{3}\right): \delta=145.0,128.4,127.4,126.0,74.3,41.3$, 19.1, 14.0. 


\section{1-Naphthalen-1-yl-ethanol (2bh) ${ }^{6 c}$}

Yield: $163.5 \mathrm{mg}$ (95\%); white solid; $\mathrm{mp} 62-63^{\circ} \mathrm{C}$.

${ }^{1} \mathrm{H} \mathrm{NMR}\left(400 \mathrm{MHz}, \mathrm{CDCl}_{3}\right): \delta=7.85-7.83(\mathrm{~m}, 1 \mathrm{H}), 7.71-7.69(\mathrm{~m}, 1 \mathrm{H})$, $7.58(\mathrm{~d}, J=8.2 \mathrm{~Hz}, 1 \mathrm{H}), 7.47(\mathrm{~d}, J=7.1 \mathrm{~Hz}, 1 \mathrm{H}), 7.34-7.25(\mathrm{~m}, 3 \mathrm{H})$, $5.34(\mathrm{q}, J=6.4 \mathrm{~Hz}, 1 \mathrm{H}), 3.41(\mathrm{~s}, 1 \mathrm{H}), 1.42(\mathrm{~d}, J=6.5 \mathrm{~Hz}, 3 \mathrm{H})$.

${ }^{13} \mathrm{C}$ NMR $\left(100 \mathrm{MHz}, \mathrm{CDCl}_{3}\right): \delta=141.7,133.9,130.4,129.0,127.9$, $126.1,125.7,125.6,123.4,122.3,66.9,24.6$.

\section{1-Naphthalen-2-yl-ethanol (2bi) $)^{17 \mathrm{f}}$}

Yield: $161.8 \mathrm{mg}$ (94\%); white solid; $\mathrm{mp} 70-71{ }^{\circ} \mathrm{C}$.

${ }^{1} \mathrm{H}$ NMR $\left(400 \mathrm{MHz}, \mathrm{CDCl}_{3}\right): \delta=7.74-7.66(\mathrm{~m}, 4 \mathrm{H}), 7.42-7.39(\mathrm{~m}, 3 \mathrm{H})$, $4.89(\mathrm{q}, J=6.5 \mathrm{~Hz}, 1 \mathrm{H}), 2.83(\mathrm{~s}, 1 \mathrm{H}), 1.46(\mathrm{~d}, J=6.5 \mathrm{~Hz}, 3 \mathrm{H})$.

${ }^{13} \mathrm{C}$ NMR $\left(100 \mathrm{MHz}, \mathrm{CDCl}_{3}\right): \delta=143.3,133.4,133.0,128.3,128.1$, 127.8, 126.2, 125.8, 123.9, 70.4, 25.2.

\section{1-Thiophen-2-ylethanol (2bj) $)^{6 c}$}

Yield: $102.1 \mathrm{mg}(91 \%)$; colorless oil.

${ }^{1} \mathrm{H} \mathrm{NMR}\left(400 \mathrm{MHz}, \mathrm{CDCl}_{3}\right): \delta=7.23(\mathrm{dd}, J=4.7,1.6 \mathrm{~Hz}, 1 \mathrm{H}), 6.98-6.95$ $(\mathrm{m}, 2 \mathrm{H}), 5.12(\mathrm{q}, J=6.4 \mathrm{~Hz}, 1 \mathrm{H}), 2.21(\mathrm{~s}, 1 \mathrm{H}), 1.59(\mathrm{~d}, J=6.4 \mathrm{~Hz}, 3 \mathrm{H})$.

${ }^{13} \mathrm{C} \mathrm{NMR}\left(100 \mathrm{MHz}, \mathrm{CDCl}_{3}\right): \delta=149.9,126.7,124.5,123.2,66.3,25.3$.

\section{1-Cyclohexylethanol (2bk) ${ }^{2 c}$}

Yield: $115.2 \mathrm{mg}(90 \%)$; colorless oil.

${ }^{1} \mathrm{H} \mathrm{NMR}\left(400 \mathrm{MHz}, \mathrm{CDCl}_{3}\right): \delta=3.54(\mathrm{p}, J=6.2 \mathrm{~Hz}, 1 \mathrm{H}), 1.87-1.83(\mathrm{~m}$, $1 \mathrm{H}), 1.78-1.74$ (m, $2 \mathrm{H}), 1.69-1.65$ (m, $3 \mathrm{H}), 1.33-1.15$ (m, $7 \mathrm{H}), 1.02-$ $0.94(\mathrm{~m}, 2 \mathrm{H})$.

${ }^{13} \mathrm{C}$ NMR $\left(100 \mathrm{MHz}, \mathrm{CDCl}_{3}\right): \delta=72.2,45.1,28.7,28.4,26.5,26.2,26.1$, 20.4 .

\section{Funding Information}

The authors thank the National Natural Science Foundation of China (21962004, 21562004), Jiangxi Provincial Department of Science and Technology (20192BAB203004), the Jiangxi Education Hall Science and Technology Foundation (GJJ180801), the emergency research project for Gannan Medical University (YJ202027) and the Fundamental Research Funds for Gannan Medical University (QD201810, QD201816) for financial support.

\section{Supporting Information}

Supporting information for this article is available online at https://doi.org/10.1055/s-0040-1707233.

\section{References}

(1) (a) Alami, M.; Hamze, A.; Provot, O. ACS Catal. 2019, 9, 3437. (b) Patel, M.; Saunthwal, R. K.; Verma, A. K. Acc. Chem. Res. 2017, 50, 240. (c) Yamamoto, Y. Chem. Soc. Rev. 2014, 43, 1575. (d) Yan, Y.; Pan, X.; Yu, X.; Wu, J. Chem. Soc. Rev. 2014, 43, 834. (e) Chinchilla, R.; Najera, C. Chem. Rev. 2014, 114, 1783. (f) Lauder, K.; Toscani, A.; Scalacci, N.; Castagnolo, D. Chem. Rev. 2017, 117, 14091. (g) Yoshida, H. ACS Catal. 2016, 6, 1799.
(2) (a) Zhang, Z.; Wu, L.; Liao, J.; Wu, W.; Jiang, H.; Li, J.; Li, J. J. Org. Chem. 2015, 80, 7594. (b) Li, J.; Li, Y.; Wang, Z.; Bian, Y.; Bai, S.; Liu, L.; Sun, J. J. Org. Chem. 2018, 83, 4257. (c) Li, L.; Herzon, S. B. J. Am. Chem. Soc. 2012, 134, 17376. (d) Liang, S.; Jasinski, J.; Hammond, G. B.; Xu, B. Org. Lett. 2015, 17, 162. (e) Gatto, M.; Belanzoni, P.; Belpassi, L.; Biasiolo, L.; Del Zotto, A.; Torantelli, F.; Zuccaccia, D. ACS Catal. 2016, 6, 7363.

(3) (a) Catalytic Cascade Reactions; Xu, P.-F.; Wang, W., Ed.; Wiley: Hoboken, 2013, 123. (b) Hayashi, Y. Chem. Sci. 2016, 7, 866. (c) Ramachary, D. B.; Jain, S. Org. Biomol. Chem. 2011, 9, 1277. (d) Climent, M. J.; Corma, A.; Iborra, S. Chem. Rev. 2011, 111, 1072. (e) Denard, C. A.; Hartwig, J. F.; Zhao, H. ACS Catal. 2013, 3, 2856 .

(4) (a) Zeng, M.; Li, L.; Seth, B. H. J. Am. Chem. Soc. 2014, 136, 7058. (b) Wu, S.; Liu, J.; Li, Z. ACS Catal. 2017, 7, 5225. (c) Ying, J.; Pu, L. J. Org. Chem. 2016, 81, 8135.

(5) Li, J.; Wang, C.; Xue, D.; Wei, Y.; Xiao, J. Green Chem. 2013, 15, 2685.

(6) (a) Schaaf, P.; Gojic, V.; Bayer, T.; Rudroff, F.; Schnerch, M.; Mihovilovic, M. D. ChemCatChem 2018, 10, 920. (b) Liu, S.; Liu, H.; Zhou, H.; Liu, Q.; Lv, J. Org. Lett. 2018, 20, 1110. (c) Liu, H.; Liu, S.; Zhou, H.; Liu, Q.; Wang, C. RSC Adv. 2018, 8, 14829.

(7) Wang, S.; Miao, C.; Wang, W.; Lei, Z.; Sun, W. ChemCatChem 2014, 6, 1612.

(8) Li, F.; Wang, N.; Lu, L.; Zhu, G. J. Org. Chem. 2015, 80, 3538.

(9) Lu, J.; Dimroth, J.; Weck, M. J. Am. Chem. Soc. 2015, 137, 12984.

(10) (a) Wang, D.-S.; Chen, Q.-A.; Lu, S.-M.; Zhou, Y.-G. Chem. Rev. 2012, 112, 2557. (b) Michon, C.; MacIntyre, K.; Corre, Y.; Agbossou-Niedercorn, F. ChemCatChem 2016, 8, 1755. (c) Wang, D.; Astruc, D. Chem. Rev. 2015, 115, 6621. (d) Chen, Y.; Pan, Y.; He, Y.-M.; Fan, Q.-H. Angew. Chem. Int. Ed. 2019, 58, 16831. (e) Yang, Z.; Luo, R.; Zhu, Z.; Yang, X.; Tang, W. Organometallics 2017, 36, 4095.

(11) Luo, N.; Liao, J.; Ouyang, L.; Wen, H.; Liu, J.; Tang, W.; Luo, R. Organometallics 2019, 38, 3025.

(12) Luo, N.; Liao, J.; Ouyang, L.; Wen, H.; Zhong, Y.; Liu, J.; Tang, W.; Luo, R. Organometallics 2020, 39, 165.

(13) Yang, Z.; Zhu, Z.; Luo, R.; Qiu, X.; Liu, J. Green Chem. 2017, 19, 3296.

(14) (a) Tachinami, T.; Nishimura, T.; Ushimaru, R.; Noyori, R.; Naka, H. J. Am. Chem. Soc. 2013, 135, 50. (b) See, J. Y.; Zhao, Y. Org. Lett. 2018, 20, 7433. (c) Liu, W.; Wang, H. Li C.-J. Org. Lett. 2016, 18, 2184.

(15) (a) Yang, Z.; Zhu, Z.; Luo, R.; Qiu, X.; Liu, J. Green Chem. 2017, 19, 3296. (b) Li, J.; Tang, W.; Ren, D.; Xu, J.; Yang, Z. Green Chem. 2019, 21, 2088. (c) Liu, J.; Yang, S.; Tang, W.; Yang, Z.; Xu, J. Green Chem. 2018, 20, 2118. (d) Talwar, D.; Wu, X.; Saidi, O.; Salguero, N. P.; Xiao, J. Chem. Eur. J. 2014, 20, 12835.

(16) Wei, Y.; Xue, D.; Lei, Q.; Wang, C.; Xiao, J. Green Chem. 2013, 15, 629.

(17) (a) Kraakman, P. A.; Nibbering, E. T. J.; De Wolf, W. H.; Bickelhaupt, F. Tetrahedron 1987, 43, 5109. (b) Ng, J. K.-P.; Li, Y.; Tan, G.-K.; Koh, L.-L.; Vittal, J. J.; Leung, P.-H. Inorg. Chem. 2005, 44, 9874. (c) Bogár, K.; Krumlinde, P.; Bacsik, Z.; Hedin, N.; Bäckvall, J.-E. Eur. J. Org. Chem. 2011, 4409. (d) Scott, D. J.; Fuchter, M. J.; Ashley, A. E. J. Am. Chem. Soc. 2014, 136, 15813. (e) Polidano, K.; Reed-Berendt, B. G.; Basset, A.; Watson, J. A.; Williams, J. M. J.; Morrill, L. C. Org. Lett. 2017, 19, 6716. (f) Swamy, P. C. A.; Varenikov, A.; Ruiter, G. Chem. Eur. J. 2020, 26, 2333. 